\title{
A NOTE ON THE ACTION OF $p$-GROUPS ON ABELIAN GROUPS
}

\section{S. PASSMAN ${ }^{1}$}

Let $p$-group $P$ act faithfully on abelian group $A$. Then $P$ also acts faithfully on $\hat{A}$, the abelian group of all linear complex characters of $A$. Suppose that all orbits in $A$ under the action of $P$ have size at most $p^{e}$ and that all orbits in $\hat{A}$ have size at most $p^{f}$. If $A$ is a $p^{\prime}$ group, then a simple application of Corollary 2.4 of [2] yields $|P|$ $\leqq p^{2 e}$ and $|P| \leqq p^{2 f}$. On the other hand, if $A$ is not a $p^{\prime}$-group, then it is not true that $|P|$ is bounded by a function of $p^{e}$ or $p^{f}$ alone. However, $|P|$ is bounded by a function of both $p^{e}$ and $p^{f}$ and we show in fact that $|P| \leqq p^{2(e+1)^{2}(f+1)^{2}}$.

We first discuss several examples.

EXAMPLE 1. Let $A$ be elementary abelian of order $p^{n+1}$ generated by $y_{0}, y_{1}, \cdots, y_{n}$ and let $P$ be elementary abelian of order $p^{n}$ generated by $x_{1}, \cdots, x_{n}$. Define the action of $P$ on $A$ by $x_{i} y_{j}=y_{j}$ if $i \neq j$ and $x_{i} y_{i}=y_{i} y_{0}$. It is easy to see that all orbit sizes in $A$ are at most $p$. On the other hand, if $\lambda \in \hat{A}$ does not contain $y_{0}$ in its kernel then $\lambda$ has $p^{n}$ conjugates. Thus $e=1$ and $f=n$ and $|P|$ is not bounded by a function of $p^{e}$.

If we consider the induced action of $P$ on $\hat{A}$, then the roles of $e$ and $f$ are reversed. Thus $f=1, e=n$ and $|P|$ is not bounded by a function of $p^{f}$.

ExAmple 2. Let $A$ be elementary abelian of order $p^{n+1}$ viewed as a vector space of dimension $n+1$ over $\mathrm{GF}(p)$. Let $P$ be a Sylow $p$ subgroup of $\operatorname{Aut}(A) \cong \mathrm{GL}(n+1, p)$. Then $|P|=p^{n(n+1) / 2}$ and $P$ can be represented as the set of all lower triangular matrices over $\mathrm{GF}(p)$ with all diagonal entries equal to 1 . This follows easily by order considerations. Let $x \in P$ and $a \in A$. Then $x a=a$ if and only if $(x-1) a=0$.

Let $a$ be fixed. Then its centralizer in $P$ is the solution space of $n$ homogeneous equations in $n(n+1) / 2$ unknowns. Thus $\left|\mathfrak{S}_{P}(a)\right|$ $\geqq p^{\{(1 / 2) n(n+1)-n\}}$ and $\left[P: \mathfrak{S}_{P}(a)\right] \leqq p^{n}$. Hence all orbits have size at most $p^{n}$ and it is easy to see that at least one orbit has size $p^{n}$. Thus $e=n$. Since $\hat{A} \cong A$ and $P$ is a Sylow $p$-subgroup of $\operatorname{GL}(n+1, p)$, it follows that also $f=n$. Therefore the exponent of $p$ in $|P|$ is essentially a quadratic function of $e$ and $f$. Since the general bound obtained in this paper is essentially biquadratic, there would appear to be room for improvement.

Received by the editors October 7, 1965.

1 This research was supported, in part, by funds from NSF Grant GP-3933. 
Now let $x$ be fixed. Then the order of its centralizer in $A$ is given by $p^{n+1-r}$ where $r$ is the rank of the matrix $(x-1)$. In particular, the elements of $P^{\#}$ (the nonidentity elements of $P$ ) with biggest centralizers in $A$ are those with rank $(x-1)=1$. If $x$ is a nonidentity central element of $P$ then $(x-1)$ contains only one nonzero entry and thus rank $(x-1)=1$. Thus in this case $P^{\#}$ contains a central element having biggest centralizer in $A$.

If $P$ does not act faithfully on $A$, then the kernel of the representation meets the center of $P$ nontrivially. Thus in this case, as in Example 2, there is a nonidentity central element $x$ of $P$ having biggest centralizer in $A$. One might expect that if $A$ is a $p$-group, then this fact is true in general. (It is, of course, clearly false if $A$ is a $p^{\prime}$ group.) If this were the case, then some simplification would occur in Lemma 7 and we could reduce the final bound to one whose exponent was cubic. However, as we see from the following example, this result is not true.

ExAmple 3. Let $A$ be elementary abelian of order $p^{2 p}$. Let $x$ and $y$ be the $(2 p) \times(2 p)$ matrices over $\mathrm{GF}(p)$ given by:

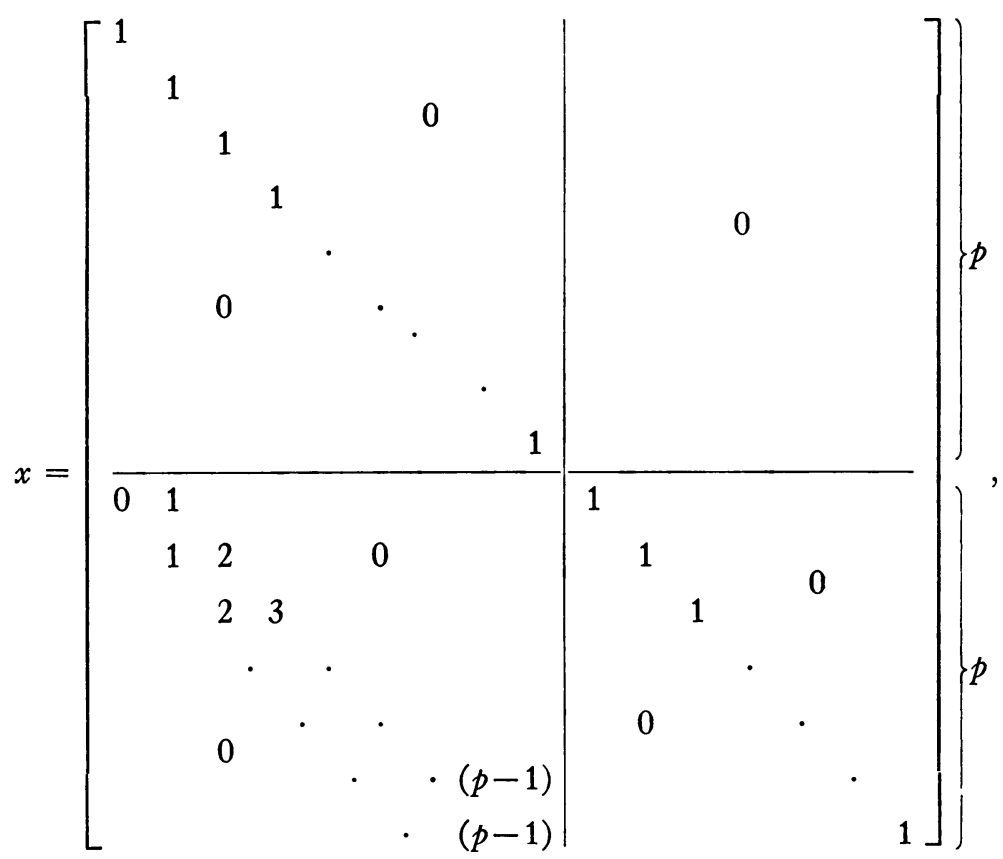




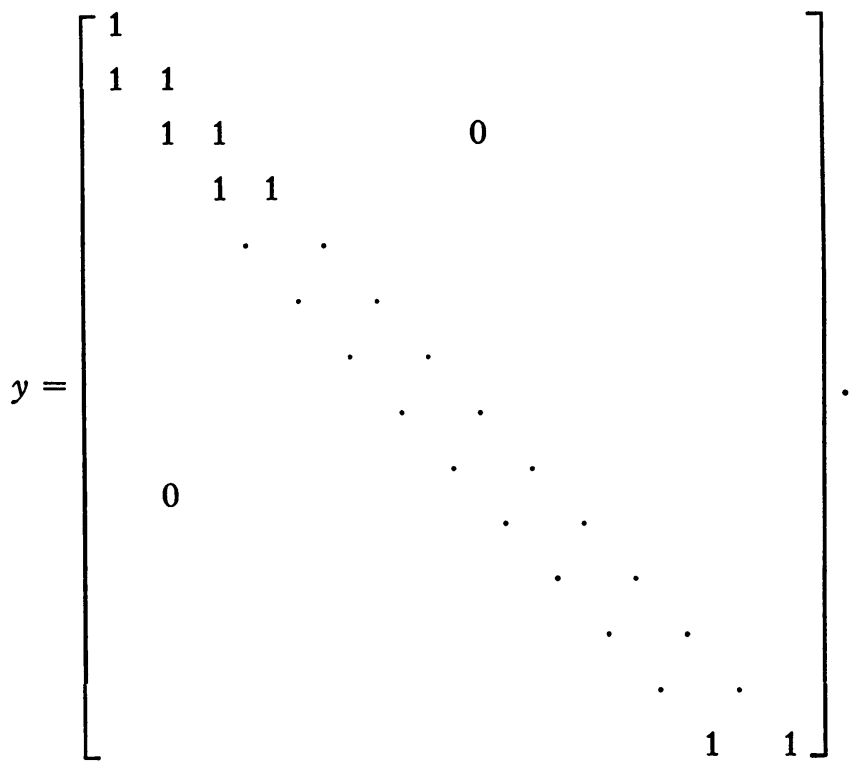

Then we see easily that

$$
x^{p}=1, \quad y^{p^{2}}=1\left(y^{p} \neq 1\right), \quad x y x^{-1}=y^{1+p} .
$$

Hence $P=\langle x, y\rangle$ is a nonabelian group of order $p^{8}$ with center $\left\langle y^{p}\right\rangle$. Now rank $(x-1)=p-1$ and rank $\left(y^{p}-1\right)=p$. Thus

$$
\left|\mathfrak{夭}_{A}(x)\right|=p^{p+1}>p^{p}=\left|\mathfrak{夭}_{A}\left(y^{p}\right)\right| \text {. }
$$

This is the required counter example.

The main result of this paper is the following.

TheOREM 4. Let p-group $P$ act on abelian p-group $A$ in such a way that all orbits in $A$ have size at most $p^{e}$ and that all orbits in $\hat{A}$, under the induced action, have size at most $p^{f}$. Then:

(i) There exists a $P$-admissible subgroup $B$ of $A$ and an element $b \in B$ such that $\mathfrak{S}_{P}(b)=\mathfrak{\complement}_{P}(B)$ and $[A: B] \leqq p^{2 e(e+1) f}$.

(ii) There exists a $P$-admissible subgroup $C$ and $A$ and $a$ linear character $\lambda \in \hat{C}$ such that $\mathfrak{夭}_{P}(\lambda)=\mathfrak{\complement}_{P}(C)$ and $[A: C] \leqq p^{2 f^{2}(f+1)}$.

In some sense part (i) is the more natural result. However, part (ii) indicates essentially how much information we can obtain from just one of the two parameters involved.

We need first a generalization of Proposition 4.7 of [1] which 
eliminates the requirement $q>f$. This result is of interest in itself and we prove it more generally than needed, by not assuming that $A$ is a $p$-group.

Lemma 5. Let $A$ be an arbitrary finite abelian group and let $q$ be the smallest prime divisor of $|A|$. Let $\mathcal{S}$ be a finite nonempty set and let $h$ be a function from $\delta$ to $A^{\#}$. Then there exists $\lambda \in \hat{A}$ such that $\left|h^{-1}(\operatorname{ker} \lambda)\right|$ $<|s| / q$.

Proof. We proceed by induction on $|A|$. Let $s \in \mathcal{S}$ and $h(s)=a$. Since $a \neq 1$ we can choose an integer $n$ so that $a^{n}$ has prime order. Define a new function $h_{1}$ by $h_{1}(s)=a^{n}$, where, of course, $n$ depends upon $s$. If $a^{n} \notin \operatorname{ker} \lambda$, then certainly $a \notin \operatorname{ker} \lambda$. Thus it suffices to assume that for each $s \in \mathcal{S}, h(s)$ has prime order.

If $A$ is not an $r$-group for some prime $r$ then $A=\cdot \sum A_{i}$ where $A_{i}$ is a Sylow $r_{i}$-subgroup of $A$. Set $S_{i}=h^{-1}\left(A_{i}\right)$. Since $h(\mathcal{S})$ contains only elements of prime order, we have $\delta=U S_{i}$. By induction we can find $\lambda_{i} \in \hat{A}_{i}$ with $\left|h^{-1}\left(\operatorname{ker} \lambda_{i}\right)\right| \leqq\left|S_{i}\right| / r_{i} \leqq\left|S_{i}\right| / q$ since $q \leqq r_{i}$ and with strict inequality if $S_{i}$ is nonempty. Set $\lambda=\prod \lambda_{i}$ so that ker $\lambda=\cdot \sum \operatorname{ker} \lambda_{i}$. Hence $\left|h^{-1}(\operatorname{ker} \lambda)\right|=\sum\left|h^{-1}\left(\operatorname{ker} \lambda_{i}\right)\right|<(1 / q) \sum\left|s_{i}\right|=(1 / q)|\delta|$ and the result follows here.

Now let $A$ be a $q$-group and let $B$ denote the subgroup of $A$ composed of all elements of order 1 and $q$. Suppose $B<A$. Since $h(\S) \subseteq B$ we can find a linear character $\mu$ of $B$ with $\left|h^{-1}(\operatorname{ker} \mu)\right|<|s| / q$. Choose $\lambda \in \hat{A}$ with $\lambda \mid B=\mu$. Then clearly $\left|h^{-1}(\operatorname{ker} \lambda)\right|<|\delta| / q$ so the result follows here.

Finally, let $A$ be an elementary abelian $q$-group. If $|A|=q$, the result is clear. Now let $|A| \geqq q^{2}$ and let $B_{0}$ be a subgroup of $A$ of index $q$ with $\left|h^{-1}\left(B_{0}\right)\right|=\beta>0$. By induction applied to the set $h^{-1}\left(B_{0}\right)$ there exists a linear character $\mu$ of $B_{0}$ with $\mid h^{-1}($ ker $\mu) \mid<\beta / q$. Let $K=\operatorname{ker} \mu$ so that $A / K$ is abelian of type $(q, q)$. Denote by $B_{0}, B_{1}$, $\cdots, B_{q}$ the $q+1$ subgroups of $A$ with $A>B_{i}>K$. Let $\alpha=\left|h^{-1}\left(A-B_{0}\right)\right|$ so that $\alpha=\sum_{1}^{a}\left|h^{-1}\left(B_{i}-K\right)\right|$. Hence for some $i \neq 0, \quad\left|h^{-1}\left(B_{i}-K\right)\right| \leqq \alpha / q$. This implies that $\left|h^{-1}\left(B_{i}\right)\right|=\left|h^{-1}(K)\right|$ $+\left|h^{-1}\left(B_{i}-K\right)\right|<\beta / q+\alpha / q$. Let $\lambda$ be a faithful character of $A / B_{i}$. Then $\operatorname{ker} \lambda=B_{i}$ and $\left|h^{-1}(\operatorname{ker} \lambda)\right|<(\beta+\alpha) / q=|s| / q$. This completes the proof.

It is easy to show that the bound in the above lemma is best possible. For example, let $A$ be elementary abelian of order $q^{n+1}$, let $\mathcal{S}=A^{\#}$ and let $h$ be the identity function. Then for all $\lambda \neq 1,\left|h^{-1}(\operatorname{ker} \lambda)\right|$ $=q^{n}-1$. Hence

$$
|s| /\left|h^{-1}(\operatorname{ker} \lambda)\right|=\left(q^{n+1}-1\right) /\left(q^{n}-1\right)<q+1 / q^{n-1} .
$$

Thus the best we can guarantee in general is $|\delta| /\left|h^{-1}(\operatorname{ker} \lambda)\right|>q$. 
Lemma 6. Let $A$ be a finite abelian group and let $q$ be the smallest prime divisor of $|A|$. Suppose p-group $P$ acts on $A$ in such a way that the orbits in $\hat{A}$ under the induced action all have size at most $p^{f}$. Let $A_{0}=A>A_{1}>\cdots>A_{n}$ be a chain of $P$-admissible subgroups of $A$ with $\mathfrak{夭}_{P}\left(A_{i}\right)<\mathfrak{\complement}_{P}\left(A_{i+1}\right)$. Then $n<f q /(q-1) \leqq 2 f$.

Proof. Set $G=A \times{ }_{\sigma} P$, the semidirect product of $A$ by $P$, so that each $A_{i}$ is normal in $G$. Set $N_{i}=\mathbb{S}_{P}\left(A_{i}\right)$. For each $i=1,2, \cdots, n$ choose $b_{i} \in N_{i}$ and $c_{i} \in A_{i-1}$ with $a_{i}=\left(b_{i}, c_{i}\right)=b_{i}^{-1} c_{i}^{-1} b_{i} c_{i} \neq 1$. These exist since by assumption $\mathfrak{C}_{P}\left(A_{i}\right)>\mathfrak{C}_{P}\left(A_{i-1}\right)$. Set $\mathcal{S}=\{1,2, \cdots, n\}$ and define function $h$ by $h(i)=a_{i}$. By Lemma 5 there exists a linear character $\lambda$ of $A$ with $\left|h^{-1}(\operatorname{ker} \lambda)\right|<n / q$.

Let $\chi$ be a constituent of $\lambda^{*}$, the induction of $\lambda$ to $G$. Since each $A_{i}$ is normal in $G$, we have $\chi \mid A_{i}=d_{i} \sum_{1}^{t_{i}} \mu_{j i}$ where the $\mu_{j_{\imath}}$ are the $t_{i}$ distinct conjugates of $\mu_{1 i}$. Now $t_{i}=\left[G: T\left(\mu_{1 i}\right)\right]$, where $T\left(\mu_{1 i}\right)$ is the inertia group of $\mu_{1 i}$ and $T\left(\mu_{1 i}\right) \supseteq A$, so $t_{i}$ is a power of $p$. Say $t_{i}=p^{s_{i}}$. Clearly $s_{0} \leqq f$.

Let $X$ be the irreducible complex representation associated with $\chi$ and let $\mathscr{L}\left(A_{i}\right)$ denote the linear space spanned by $\boldsymbol{X}\left(A_{i}\right)$. Clearly $\mathfrak{L}\left(A_{i}\right) \supseteq \mathfrak{L}\left(A_{i+1}\right)$ and $\operatorname{dim} \mathfrak{L}\left(A_{i}\right)=t_{i}$. If $a_{i} \notin \operatorname{ker} \lambda$, then $a_{i} \notin \operatorname{ker} \chi$. Thus $\boldsymbol{X}\left(N_{i}\right)$ centralizes $\mathscr{L}\left(A_{i}\right)$ but not $\mathscr{L}\left(A_{i-1}\right)$ and so $s_{i-1}>s_{i}$. Since $s_{0} \leqq f$ and $s_{n} \geqq 0$, it follows that $\left|\delta-h^{-1}(\operatorname{ker} \lambda)\right| \leqq f$. Then

$$
n=|\delta|=\left|\delta-h^{-1}(\operatorname{ker} \lambda)\right|+\left|h^{-1}(\operatorname{ker} \lambda)\right|<f+n / q .
$$

Thus $n<f q /(q-1) \leqq 2 f$.

We now assume that the hypotheses of Theorem 4 are satisfied.

Lemma 7. (i) Suppose that for all $a \in A, \mathfrak{S}_{P}(a)>\{1\}$. Then there exists a nonidentity normal subgroup $M_{1}$ of $P$ with $\left[A: \mathfrak{E}_{A}\left(M_{1}\right)\right]$ $\leqq p^{e(e+1)}$. (ii) Suppose that for all $\lambda \in \hat{A}, \mathfrak{夭}_{P}(\lambda)>\{1\}$. Then there exists a nonidentity normal subgroup $M_{2}$ of $P$ with $\left[A: \mathfrak{S}_{A}\left(M_{2}\right)\right] \leqq p^{f(f+1)}$.

Proof. We prove both parts at the same time. Thus we consider $P$ acting on a group $B$ in such a way that all orbits have size at most $p^{d}$. Here $B=A$ and $d=e$ for Part (i) and $B=\hat{A}$ and $d=f$ for Part (ii).

It clearly suffices to assume that $p^{d}$ is in fact the maximum orbit size. If $|P| \leqq p^{d}$, then certainly there exists $b \in B$ with $\mathfrak{E}_{P}(b)=\{1\}$. Thus $|P| \geqq p^{d+1}$ and we can choose $N$ normal in $P$ with $|N|=p^{d+1}$. Let $J=\left\{x_{i}\right\}$ contain one generator for each subgroup of $N$ of order $p$. Then $|\Im| \leqq\left(p^{d+1}-1\right) /(p-1)<p^{d+1}$. Let $b \in B$. Since $\mathfrak{\Xi}_{P}(b)>\{1\}$, it follows that for some $i, x_{i} \in \mathfrak{C}_{P}(b)$ so that $b \in \mathfrak{C}_{B}\left(x_{i}\right)$. Thus $B=U \mathfrak{S}_{B}\left(x_{i}\right)$. Choose $y \in J$ with $\left|\mathfrak{\Im}_{B}(y)\right| \geqq\left|\mathfrak{\Im}_{B}\left(x_{i}\right)\right|$ for all $x_{i} \in J$. Then counting elements in the above union, we have $|B|<\left|\mathfrak{夭}_{B}(y)\right| p^{d+1}$. Since $B$ is a p-group, this yields $|B| \leqq\left|\mathfrak{S}_{B}(y)\right| p^{d}$. 
If $B=A$, then $\left[A: \mathfrak{S}_{A}(y)\right] \leqq p^{d}$. Now let $B=\hat{A}$. Since $A$ is abelian, the map $a \rightarrow a^{-1} y(a)$ is an endomorphism. The kernel is clearly $\mathfrak{S}_{A}(y)$ and we denote the image by $(y, A)$. If $\lambda \in \hat{A}$, then $\lambda^{y}=\lambda$ if and only if $\operatorname{ker} \lambda \supseteq(y, A)$. Thus $\left|\mathfrak{\mho}_{\hat{A}}(y)\right|=[A:(y, A)]=\left|\mathfrak{\mho}_{A}(y)\right|$ and hence $\left[A: \mathfrak{S}_{A}(y)\right] \leqq p^{d}$ in this case also.

Let $M=y^{P}$. Then $M$ is normal in $P, M \neq\{1\}$ and $M \subseteq N$. Hence $|M| \leqq p^{d+1}$ and $M=\left\langle y_{1}, \cdots, y_{j}\right\rangle$ where the $y_{i}$ are $j \leqq d+1$ conjugates of $y$. Clearly $\left[A: \mathfrak{E}_{A}\left(y_{i}\right)\right] \leqq p^{d}$ and since $\mathfrak{E}_{A}(M)=\cap \mathfrak{E}_{A}\left(y_{i}\right)$ we obtain finally $\left[A: \mathfrak{S}_{A}(M)\right] \leqq p^{d(d+1)}$.

We now proceed with the proof of Theorem 4. The proofs of Parts (i) and (ii) differ only in which part of the above lemma we apply. Thus we will consider only Part (ii). We show by induction on $|P|$ that there exists a chain of $P$-admissible subgroups $A_{0}=A>A_{1}>A_{2}$ $>\cdots>A_{n}$ with $\left[A_{i}: A_{i+1}\right] \leqq p^{f(f+1)}$ and $\mathfrak{G}_{P}\left(A_{i}\right)<\mathfrak{E}_{P}\left(A_{i+1}\right)$. In addition there exists $\lambda \in \hat{A}_{n}$ with $\mathfrak{C}_{P}(\lambda)=\mathfrak{C}_{P}\left(A_{n}\right)$.

If $P$ does not act faithfully then $K=\mathfrak{夭}_{P}(A)>\{1\}$ and we apply induction to $P / K$. Thus it suffices to assume that $P$ is faithful. If there exists $\lambda \in \hat{A}$ with $\mathfrak{S}_{P}(\lambda)=\{1\}$ then we can take $A_{n}=A_{0}$ and the result follows. Thus we can assume that for all $\lambda \in \hat{A}, \mathfrak{\complement}_{P}(\lambda)>\{1\}$. Therefore by Lemma 7 (ii) there exists nonidentity normal subgroup $M$ of $P$ with $\left[A: \mathfrak{G}_{A}(M)\right] \leqq p^{f(f+1)}$. Set $A_{1}=\mathfrak{\complement}_{A}(M)$ so that $\mathfrak{\complement}_{P}\left(A_{1}\right) \supseteq M$ $>\{1\}=\mathfrak{S}_{P}\left(A_{0}\right)$. Now $P / M$ acts on $A_{1}$. If $\mu \in \hat{A}_{1}$ then there exists $\lambda \in \hat{A}$ with $\lambda \mid A_{1}=\mu$. Thus the conjugates of $\mu$ are the restrictions of conjugates of $\lambda$. Hence all orbits in $\hat{A}_{1}$ under the action of $P / M$ have size at most $p^{f}$. Clearly this result follows by induction.

Now by Lemma $6, n<f p /(p-1) \leqq 2 f$. Thus if we set $C=A_{n}$, the theorem is proved.

We now turn to the results discussed in the introduction.

THEOREM 8. Let p-group $P$ act faithfully on abelian group ${ }^{\prime \prime} A$ in such $a$ way that all orbits in $A$ have size at most $p^{e}$ and that all orbits in $\hat{A}$, under the induced action, have size at most $p^{f}$.

(i) If $A$ is a $p^{\prime}$-group, then $|P| \leqq p^{2 e}$ and $|P| \leqq p^{2 f}$.

(ii) In any case $|P| \leqq p^{2(e+1)^{2}(f+1)^{2}}$.

Proof. Suppose first that $A$ is a $p^{\prime}$-group. By Corollary 2.4 (iii) of [2] there exists $a \in A$ with $\left|\mathfrak{C}_{P}(a)\right| \leqq|P|^{1 / 2}$. Thus $p^{e} \geqq\left[P: \mathfrak{\complement}_{P}(a)\right]$ $\geqq|P|^{1 / 2}$ and $p^{2 e} \geqq|P|$. Since $P$ also acts faithfully on $\hat{A}$ we get $p^{2 f} \geqq|P|$.

Now let $A$ be an arbitrary finite abelian group. Then $A=A_{1}+A_{2}$ where $A_{1}$ is its Sylow $p$-subgroup and $A_{2}$ is its Hall $p^{\prime}$-subgroup. Let $K=\mathfrak{C}_{P}\left(A_{1}\right)$. Then $K$ is normal in $P$ and $P / K$ acts faithfully on $A_{1}$. Since $K$ acts faithfully on $A_{2}$ we have $|K| \leqq p^{2 e}$ and $|K| \leqq p^{2 f}$. 
We apply Theorem 4 to the action of $P / K=P_{1}$ on $A_{1}$. Let $N=\mathfrak{S}_{P_{1}}(B)$ or $N=\mathbb{E}_{P_{1}}(C)$ according to which part of that theorem we apply. Let $\left[A_{1}: \mathfrak{S}_{A_{1}}(N)\right]=p^{2}$. Then $z \leqq 2 e(e+1) f$ from Part (i) and $z \leqq 2 f^{2}(f+1)$ from Part (ii). Now $A_{1} / \mathbb{S}_{A_{1}}(N)$ has $z$ generators $\left\{a_{i}\right\}$ in $A_{1}$ and since $\left[N: \mathfrak{G}_{N}\left(a_{i}\right)\right] \leqq p^{e}$ we see that $\left[N: \mathfrak{E}_{N}\left(A_{1}\right)\right] \leqq p^{z e}$. Hence $|N| \leqq p^{2 e}$ since $N$ acts faithfully. Finally since $N$ is the centralizer of an element, $\left[P_{1}: N\right] \leqq p^{e}$ from Part (i) and $\left[P_{1}: N\right] \leqq p^{f}$ from Part (ii). Thus

$$
\log _{p}|P| \leqq 2 e(e+1) f e+e+2 e,
$$

and

$$
\log _{p}|P| \leqq 2 f(f+1) f e+f+2 f .
$$

Certainly the smaller of these two bounds is less than $2(e+1)^{2}(f+1)^{2}$. Thus the result follows.

Applying the above to each Sylow subgroup of $G$ we obtain the following.

COROLlARY 9. There exists an integer valued function $k$ with the following property. If group $G$ acts faithfully on abelian group $A$, then $|G| \leqq k(m, n)$, where $m$ is the maximal orbit size in $A$ and $n$ is the maximal orbit size in $\hat{A}$ under the induced action.

\section{REFERENCES}

1. I. M. Isaacs and D. S. Passman, $A$ characterization of groups in terms of the degrees of their characters, Pacific J. Math. 15 (1965), 877-903.

2. D. S. Passman, Groups with normal, solvable Hall p'-subgroups, Trans. Amer. Math. Soc. (to appear).

University of California, Los Angeles 